prednisone-equivalent $\leq 7.5 \mathrm{mg} / \mathrm{day}$, and stable maintenance doses of immunosuppressants). A pre-defined subset of patients was also evaluated, with high disease activity (HDA: SLEDAI$2 \mathrm{~K} \geq 10$ at Screening). Differences in clinical response between patients treated with atacicept and $\mathrm{PBO}$ at Week 24 were analysed using odds ratio estimated from logistic regression.

Results The ITT population included 306 patients, and 158 had HDA. There was a trend towards improved SRI-4 response with atacicept vs $\mathrm{PBO}$ at Week $24(\mathrm{p}=\mathrm{ns}$ in primary analysis; screening visit as baseline, BL). In a pre-specified sensitivity analysis using study day 1 as BL, a significantly larger proportion of patients on atacicept achieved SRI-4 response at week 24. In the HDA subpopulation, there were significant improvements in SRI-4,-5, $-6,-7$ and -8 response rates and attainment of LDA with atacicept $150 \mathrm{mg}$ vs PBO (table 1). Atacicept was associated with increased serum C3 and C4, and decreased IgG, IgA, IgM and anti-dsDNA antibodies over time. Rates of treatment emergent adverse event (TEAE) and serious TEAEs were similar among groups. The most frequent serious TEAEs were infections but the incidence was not increased in the atacicept groups vs PBO.

Conclusions Atacicept showed evidence of efficacy in SLE with a dose-dependent reduction of SLE disease activity in patients with HDA. Atacicept was associated with an acceptable safety profile. These results also suggest that more discriminatory endpoints will be useful for future SLE clinical trials.

\section{S7A:6 BASELINE SERUM LEVELS OF BAFF OR APRIL ARE INDEPENDENT PREDICTORS OF SLEDAI RESPONSE AFTER 12 MONTHS OF TREATMENT WITH BELIMUMAB IN PATIENTS WITH REFRACTORY SYSTEMIC LUPUS ERYTHEMATOSUS}

${ }^{1} S$ Piantoni, ${ }^{1} \mathrm{~L}$ Andreoli, ${ }^{2} \mathrm{~T}$ Lowin, ${ }^{1} \mathrm{R}$ Kumar, ${ }^{1} \mathrm{~F}$ Regola, ${ }^{1} \mathrm{P}$ Airò, ${ }^{1} \mathrm{~F}$ Franceschini, ${ }^{1}$ A Tincani, ${ }^{2} \mathrm{G}$ Pongratz. ${ }^{1}$ Rheumatology and Clinical Immunology, Spedali Civili and University of Brescia, Italy; ${ }^{2}$ Rheumatology Department and Hiller Research Centre for Rheumatology, University Hospital Düsseldorf, Germany

\subsection{6/lupus-2018-abstract.43}

Background Belimumab, a monoclonal antibody targeting BlyS (B lymphocyte stimulator), is used in refractory Systemic Lupus Erythematosus (SLE). Pivotal clinical trials showed that SLE patients with positive anti-dsDNA antibodies and reduced levels of C3 and/or C4 fractions were those more likely to be responders to treatment. Our study aims at exploring predictors of response to Belimumab in the post-marketing experience in consecutive SLE patients treated at a single centre.

Methods Twenty-one patients received Belimumab intravenously at standard regimen $(10 \mathrm{mg} / \mathrm{kg}$ at $0-15-30$ days and then every 4 weeks). Anti-dsDNA were tested by Farr assay and $\mathrm{C} 3 / \mathrm{C} 4$ levels by nephelometry. Biomarkers belonging to the TNF superfamily and related to B cell activity (BAFF, APRIL, sBCMA, sCD40L, sTACI, TWEAK) were tested by ELISA. All laboratory parameters were tested at baseline and every 6 months afterwards. SLE disease activity was assessed by SLEDAI-2K score. General linear modelling and correlation analysis were performed using SPSS.

Results Enrolled patients were 2 males and 19 females with a median (25th-75th percentile) age of 38 (31-42) years. The disease duration at time of Belimumab start was $12(8-19)$ years. The baseline SLEDAI score was 6 (4-9), the antidsDNA level was $26(11-99) \mathrm{UI} / \mathrm{ml}$, and their C3 and C4 level was 72 (56-86) and 9 (7-15) $\mathrm{mg} / \mathrm{dL}$, respectively.

All the parameters of the TNF superfamily showed moderate/strong correlation ( $\mathrm{r}$ values ranging from 0.543 and 0.989 , $\mathrm{p}<0.01$ ). With and without correction for different variables, BAFF and APRIL serum levels measured at the start of Belimumab treatment were the most robust predictors of relative SLEDAI reduction after 12 months of treatment (table 1).

In contrast, C3, C4, anti-dsDNA, and SLEDAI were less likely to predict relative SLEDAI change at 12 month of Belimumab treatment (uncontrolled model: C3 p=0.410; C4 $\mathrm{p}=0.778$; anti-dsDNA $\mathrm{p}=0.412$ ) in this cohort of patients preselected for the treatment with Belimumab.

Conclusions In this preselected 'real-life' cohort of refractory SLE patients fulfilling the requirements for Belimumab treatment baseline serum levels of BAFF or APRIL are independent predictors of response to treatment. Therefore, BAFF and APRIL could be useful for response estimation in patients qualifying for Belimumab treatment.

\section{S7A:7 ADMINISTRATION OF SERPINB3 DELAYS GLOMERULONEPHRITIS AND ATTENUATES THE LUPUS- LIKE DISEASE IN LUPUS MURINE MODELS BY AN IMMUNOMODULATORY EFFECT}

${ }^{1} \mathrm{M}$ Gatto, ${ }^{2} \mathrm{~L}$ Cavicchioli, ' $\mathrm{R}$ Luisetto, ${ }^{3} \mathrm{G}$ Codolo, ${ }^{1} \mathrm{G}$ Maggioni, ${ }^{1} \mathrm{~F}$ Saccon, ${ }^{1} \mathrm{M}$ Beggio, ${ }^{4} \mathrm{P}$ Pontisso, ${ }^{1} \mathrm{~A}$ Ghirardello, ${ }^{1} \mathrm{~A}$ Doria. ${ }^{1}$ University of Padova - Unit of Rheumatology, Department of Medicine, Padova, Italy; ${ }^{2}$ University of Padova - Department of Biomedicine and Nutrition, Padova, Italy; ${ }^{3}$ University of Padova - General Pathology Unit, Department of Biology, Padova, Italy; ${ }^{4}$ University of Padova - Department of Clinical and Experimental Medicine, Padova, Italy

\subsection{6/lupus-2018-abstract.44}

Background Abnormal apoptosis and clearance of cellular debris concur to development of systemic lupus erythematosus (SLE). SERPINS (serin-protease inhibitors) are ancient molecules regulating immune homeostasis. SERPINB3 modulates apoptosis and is hypoexpressed on SLE B cells.

Aim To explore the effects of SERPINB3 administration in murine lupus models, focusing on glomerulonephritis.

Abstract S7A:6 Table 1 General linear modelling to calculate predictive value of baseline BAFF and APRIL levels for the relative change of SLEDAI at 12 month

\begin{tabular}{|l|c|c|}
\hline $\begin{array}{l}\text { General linear modeling (GLM), } \\
\text { ANOVA }\end{array}$ & $\begin{array}{c}\text { Baseline APRIL as predictor of SLEDAI } \\
\text { change at 12 month BELIMUMAB }\end{array}$ & $\begin{array}{c}\text { Baseline BAFF as predictor of SLEDAI } \\
\text { change at 12 month BELIMUMAB }\end{array}$ \\
\hline uncontrolled & $F=8.289 ; p<0.001$ & $F=8.195 ; p<0.001$ \\
\hline $\begin{array}{l}\text { controlled for initial AGE, } \\
\text { LEUCOCYTES, CRP }\end{array}$ & $F=7.272 ; p=0.007$ & $F=7.647 ; p=0.006$ \\
\hline $\begin{array}{l}\text { controlled for initial AGE, } \\
\text { LEUCOCYTES, CRP, SLEDAI }\end{array}$ & $F=5.929 ; p=0.019$ & $F=6.624 ; p=0.015$ \\
\hline
\end{tabular}


Mantel-Cox: $p=0.002$

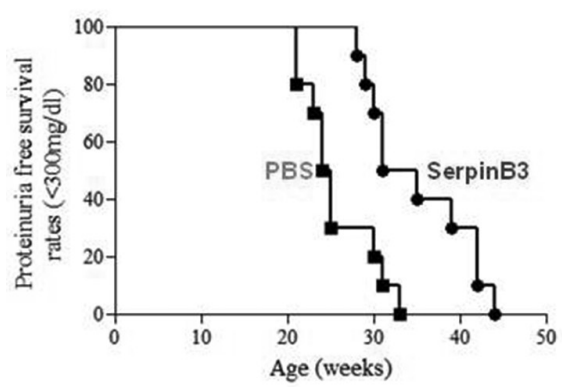

Mantel-Cox: $p=0.014$

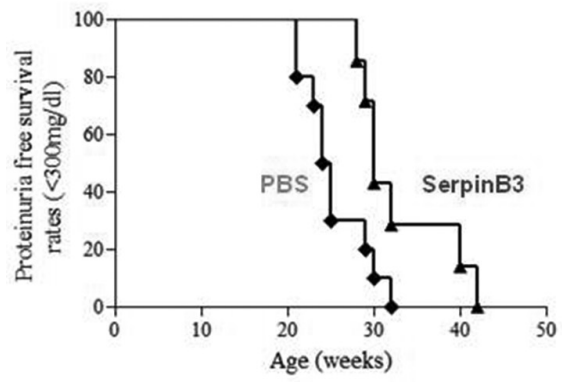

Mantel-Cox: $p=0.001$

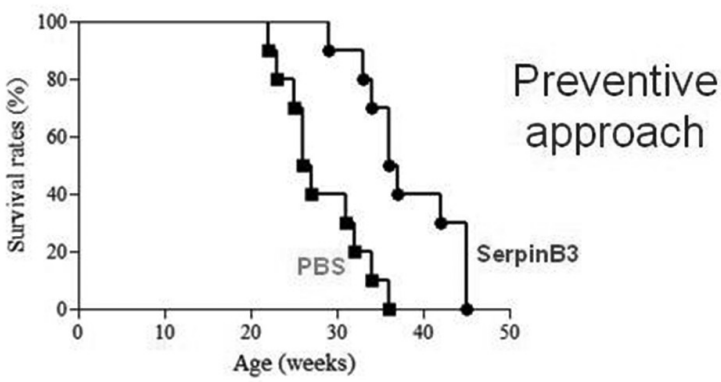

Mantel-Cox: $p=0.009$

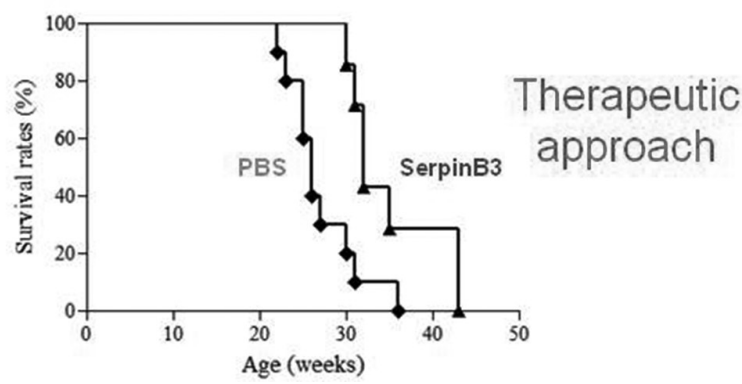

Abstract S7A:7 Figure 1 Survival in NZB/W F1 brought to natural death

Methods NZB/W F1 and MRL/lpr mice were used. 40 NZB/W F1 mice were divided into 4 groups of 10 mice each and intraperitoneally injected twice a week starting before occurrence of proteinuria traces (group 1 and 2, prophylactic approach) or after development of proteinuria $30 \mathrm{mg} / \mathrm{dl}$ (group 3 and 4, therapeutic approach) with hrSERPINB3 $(7.5 \mu \mathrm{g} / 0.1 \mathrm{~mL}$ prophylactic approach, or $15 \mu \mathrm{g} / 0.1 \mathrm{~mL}$ therapeutic approach) or PBS $(0.1 \mathrm{~mL}) .20 \mathrm{MRL} / \mathrm{lpr}$ mice were injected with hrSERPINB3 (group 5, n=10) or PBS (group $6, \mathrm{n}=10$ ) with a prophylactic approach. We assessed time of occurrence and titers of antidsDNA and anti-C1q antibodies by ELISA; proteinuria and serum creatinine; overall- and proteinuria-free survival. Six NZB/W F1 mice were sacrificed at week 27 , while $10 \mathrm{MRL} / \mathrm{lpr}$ mice at week 13 and another 10 at 16/18 weeks for histological kidneys comparison. Flow-cytometry was performed on MRL/lpr splenocytes.

Non parametric tests were performed for statistics; proteinuria-free $(<300 \mathrm{mg} / \mathrm{dl})$ and overall survival were evaluated by Kaplan-Meier method.

Results Levels of autoantibodies were significantly decreased and delayed in group 1 vs group 2, group 3 vs group 4, and group 5 vs group 6 ( $\mathrm{p}<0.0001$ for all). Proteinuria levels were significantly reduced and proteinuria-free and overall survival were significantly improved in SERPINB3 groups vs controls (figure 1). No differences were found among creatinine serum levels. Histological analysis showed a lower prevalence of severe tubular lesions in group 5 vs group $6 \mathrm{MRL} / \mathrm{lpr}$ mice at week 16 (chi-squared $\mathrm{p}=0.014$ ), and mice belonging to SERPINB3 groups showed a trend toward a reduced prevalence of severe glomerular and tubular lesions. Th17:Treg ratio significantly decreased due to a remarkable increase in Treg levels in MRL/lpr mice treated with SERPINB3.

Conclusions Administration of SERPINB3 significantly improves disease and delays the onset of severe glomerulonephritis in lupusprone mice. SERPINB3 may influence immune-cell function through immunoregulatory effects involving promotion of Treg.
S7A:8 EFFICACY AND SAFETY OF USTEKINUMAB, AN INTERLEUKIN 12/23 INHIBITOR, IN PATIENTS WITH ACTIVE SYSTEMIC LUPUS ERYTHEMATOSUS: RESULTS OF A PHASE 2, RANDOMISED PLACEBO-CONTROLLED STUDY

${ }^{1} \mathrm{R}$ van Vollenhoven, ${ }^{2} \mathrm{~B}$ Hahn, ${ }^{3} \mathrm{G}$ Tsokos, ${ }^{4} \mathrm{C}$ Wagner, ${ }^{5} \mathrm{P}$ Lipsky, ${ }^{4} \mathrm{~B}$ Hsu, ${ }^{4} \mathrm{M}$ Chevrier, ${ }^{4} \mathrm{R}$ Gordon, ${ }^{6} \mathrm{M}$ Triebel, ${ }^{4} \mathrm{~S}$ Rose. ${ }^{7}$ Amsterdam Rheumatology and Immunology Centre ARC, Amsterdam, The Netherlands; ${ }^{2}$ University of California Los Angeles, USA; ${ }^{3}$ Beth Israel Hospital, Boston, USA; ${ }^{4}$ Janssen Research and Development, LLC, Spring House, USA; ${ }^{5}$ AMPEL BioSolutions, LLC, Charlottesville, USA; ${ }^{6}$ Janssen Biologics Europe, Leiden, The Netherlands

\subsection{6/lupus-2018-abstract.45}

Purpose IL12 and IL23 pathway have been linked to SLE pathogenesis. Anti-IL12/23 monoclonal antibody ustekinumab (UST) previously approved for psoriasis, psoriatic arthritis, and Crohn's diseasewas evaluated in pts with active SLE.

Methods A Ph2, PBO-controlled study in 102 adults with seropositive SLE by SLICC criteria with active disease despite standard-of-care therapy were randomised to UST vs PBO, added to standard care. Primary endpoint was the proportion achieving SLE response index (SRI)-4 response at wk24. Secondary endpoints included change SLEDAI-2K, Physician's Global Assessment (PGA), and BICLA response.

Results The mITT population (includes pts who received at least one dose) at wk24 revealed $60 \%$ of UST pts with SRI-4 response vs $31 \%$ in $\mathrm{PBO}(\mathrm{p}=0.0046)$. Pts in UST group had greater median change from wk0 to wk24 in SLEDAI-2K and PGA vs PBO (table 1). No difference was observed in BICLA composite response at wk24, but more UST pts had no BILAG worsening vs PBO. Through wk24, 78\% of UST pts and $67 \%$ of $\mathrm{PBO}$ pts had greater than or equal to $1 \mathrm{AE}$; $8.3 \%-9.5 \%$, respectively, had greater than or equal to $1 \mathrm{SAE}$ (table 1). There were no deaths in the study. Safety events 